\title{
EVALUATION OF TWO ROUTINELY USED 25OHD ASSAYS AND SERUM VARIABLES IN PATIENTS ON DIALYSES
}

\section{Z. Lőcsei ${ }^{1}$, L. Kovács ${ }^{1}$, D. Balogh ${ }^{5}$, A. Szijártó ${ }^{5}$ and B. Kálmán ${ }^{2}$, G.L. Kovács ${ }^{4,5}$, E. Toldy ${ }^{3,5}$}

$1^{\text {st }}$ Department of Medicine ${ }^{1}$, Center for Molecular Medicine ${ }^{2}$, Central Laboratory ${ }^{3}$ Markusovszky Teaching Hospital of County Vas, Szombathely and Institute of Laboratory Medicine ${ }^{4}$, Institute of Diagnostics ${ }^{5}$, University of Pécs, Hungary

\section{Background}

The total 25-hydoxy-vitamin-D (t-25OHD) level can be routinely assessed by various methods and reflects vitamin D intake. Results are influenced by the serum variables affected by dialyses.

Aims: to examine t-25OHD and bioavailable vitamin D (bio25OHD) by two most frequently used methods in patients on peritoneal- (PD) and hemodialysis (HD).

\section{Investigated cases}

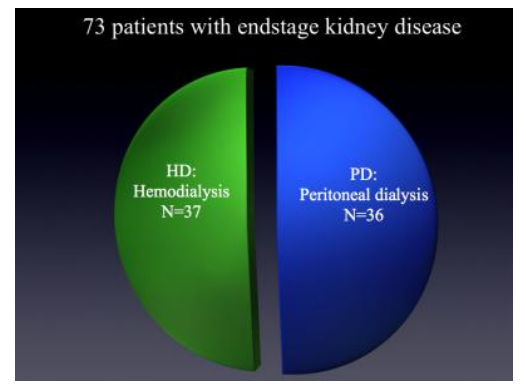

We studied $37 \mathrm{HD}(64 \pm 15$ years, 17 females, 20 males $)$ and 36 PD (63 \pm 18 years, 15 females, 21 males) patients without vitamin D substitution.

\section{Methods}

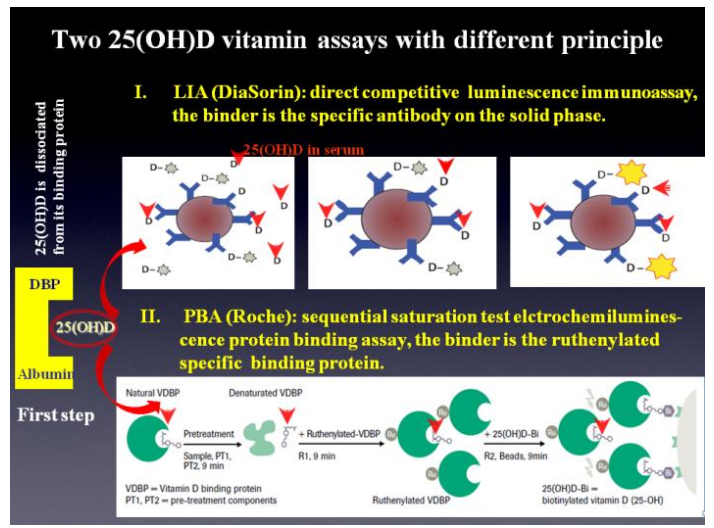

All 73 sera were analyzed for $\mathbf{t}-\mathbf{2 5 O H D}$ by two assays with different principles.

In addition the following biomolecules were measured: PTHbiointact (Bio-PTHi) by immunometric assay (ECLMA, Roche), vitamin D binding protein (DBP) by turbidimetry (Dako), and total protein (TP), albumin (Alb) and calcium (Ca) by colorimetry (Roche, Modular).

The bio-25OHD values were calculated (Vermeulen et al. 1999, Bhan et. al. 2012) The cutoff values for evaluation of Vitamin D supply or $\mathrm{t}-25 \mathrm{OHD}$ and bio-25OHD are summarized in the table.

\begin{tabular}{|l|c|c|}
\hline \multirow{2}{*}{$25 \mathrm{OHD}$ fractions } & \multicolumn{2}{|c|}{ Optimal (nmol/L) } \\
\cline { 2 - 3 } & LIA & PBA \\
\hline t-25OHD & $>75$ & $>75$ \\
\hline bio-25OHD & $>6.1^{*}$ & $>6.4^{*}$ \\
\hline
\end{tabular}

\section{Results}

The frequency of undetectable t-25OHD level was higher by PBA $(29 \%)$ than by LIA $(1.4 \%)$.

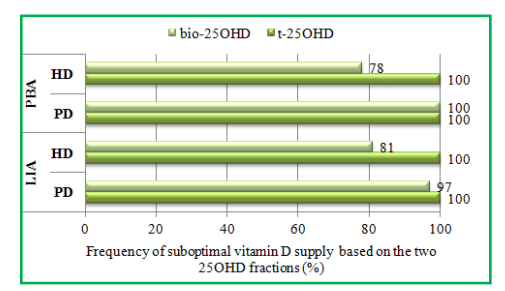

All investigated cases had suboptimal vitamin D supply by both t$25 \mathrm{OHD}$ assays. However, the frequency of suboptimal supply decreased on the bases of bio-25OHD levels especially in HD.

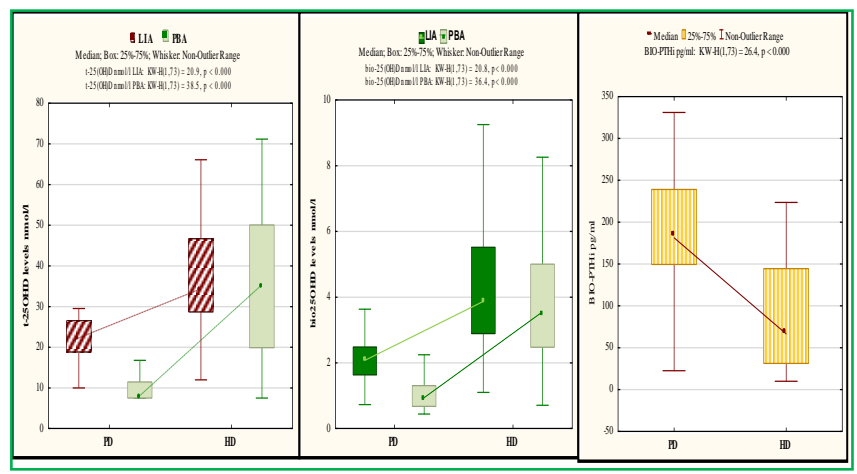

Values of t- and bio-25OHD were significantly lower in PD than in $\mathrm{HD}$, while Bio-PTHi levels were significantly lower in HD than in PD.

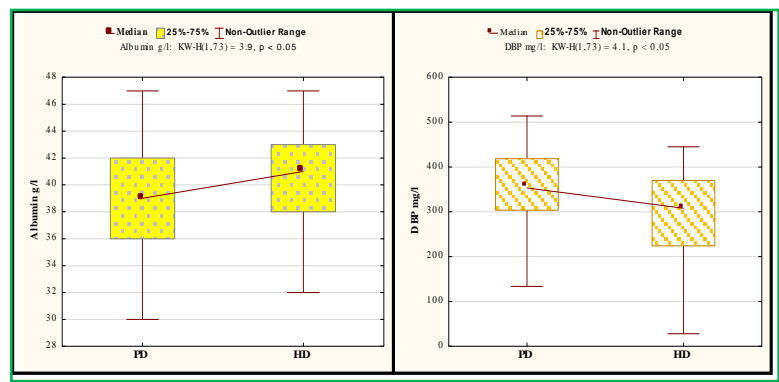

Albumin levels were lower in PD than in HD, but DBP levels were higher in PD than in HD ( $\mathrm{p}<0.05)$.

Positive correlations were observed with both methods between t25OHD and albumin levels in PD only (PBA: $r=0.36$; $p<0.05$; LIA: $\mathrm{r}=0.48 ; \mathrm{p}<0.01$ ).

Negative correlations were observed between Bio-PTHi and t25OHD levels (PBA: $r=-39$, LIA: $r=-0.42 ; p<0.05$ ) in HD only; negative correlations observed between Bio-PTHi and bio-25OHD were similar in both HD and PD (LIA: PD $r=-0.40$, HD $r=-0.54$, $\mathrm{p}<0.01$; PBA: PD $\mathrm{r}=-0.49$, HD r=-0.44).

The correlations of $\mathrm{t}-25 \mathrm{OHD}$ levels assessed by LIA and PBA were different (HD: $r=0.89 ; p<0.001 ; P D: r=0.47, p<0.01$ ), but correlations of bio-25OHD values were similar in both groups (HD: $r=0.85$; PD: $\mathrm{r}=0.83, \mathrm{p}<0.001$ ).

\section{Conclusions}

- Assessment of vitamin D supply by LIA and PBA is influenced by lower albumin levels especially in PD.

- Estimation of bio-25OHD is more suitable in PD, while t$25 \mathrm{OHD}$ is a reliable measure with either method in HD. 